\title{
Las TICS y su utilización para la lectura, la lingüística y la literatura como Tools generadoras de competencias en los discentes del milenio
}

\section{ICTs and their use for reading, linguistics and literature as tools that generate skills in the millennial students}

María Fernanda Moreira Macías

Universidad Agraria del Ecuador, Ecuador

Autor para correspondencia: fmmva_delfin@hotmail.com

Fecha de recepción: 15 de Septiembre de 2016 - Fecha de aceptación: 05 de Noviembre de 2016

Resumen: ¿Por qué existe el Analfabetismo funcional en los estudiantes actuales? De acuerdo a investigaciones realizadas se ha demostrado que los estudiantes presentan muchas falencias al exponer o sustentar sus trabajos desarrollados en las aulas de clases y de igual situación y similitud ocurre al momento de expresar sus ideas. ¿Es importante el uso de las Tics en la metodología de la enseñanza? Las nuevas reformas curriculares nos obliga, tanto a docentes como estudiantes a utilizar las Tics como herramientas en el proceso enseñanza-aprendizaje, pero cuando estas son mal utilizadas, generan el desarrollo de la Pobreza Lingüística, de la lectura y de la literatura en los estudiantes universitarios, razón por la cual se ha llevado a cabo esta investigación, que tiene como objetivo analizar el uso de las Tics y su incidencia en el proceso académico, para ello se analizan los diferentes escenarios en donde se desenvuelven el proceso enseñanza-aprendizaje. La metodología empleada para obtener la información está fundamentada en la observación y en las técnicas de la encuesta, la misma que se ha realizado a 100 estudiantes colegiales y 300 estudiantes de diferentes universidades de la ciudad de Guayaquil y una entrevista a 30 docentes de las mismas universidades. La investigación es descriptiva y analítica correlacional permitiendo establecer la relación que existe entre el ambiente de aprendizaje, la utilización de las Tics, la implementación de la lectura, la literatura y el desarrollo de la lingüística. El trabajo realizado ha permitido establecer la importancia de los ambientes de aprendizaje, los niveles de aceptación de las Tics por parte de los docentes y estudiantes, su uso y repercusión en las habilidades comunicativas.

Palabras claves: lingüística; tics; analfabetismo funcional; tools; competencias; discentes

Abstract: Why is the functional illiteracy in today's students? According to research conducted has shown that students have many flaws to expose or support their work done in the classroom and in the same situation and similarity it occurs when expressing their ideas.

How important is the use of ICT in teaching methodology? The new curriculum reform requires us, both teachers and students to use ICTs as tools in the teaching-learning process, but when they are misused, generate the development of linguistics Poverty, reading and literature in students university, reason why it has conducted this research, which aims to analyze the use of ICT and its impact on the academic process, to do the different scenarios where the teaching-learning process unfold analyzed. The methodology used to obtain information is based on observation and technical survey, it has been made 100 college students and 300 students from different universities in the city of Guayaquil and an interview with 30 teachers of the same universities. The research 
is descriptive and correlational analytical possible to establish the relationship between the learning environment, the use of ICTs, implementation of reading, literature and development of linguistics. The work has established the importance of learning environments, levels of acceptance of ICTs by teachers and students, their use and impact on communication skills.

Key words: linguistics; tics; functional illiteracy; tools; skills; learners

\section{Antecedentes}

Desde que existe la humanidad nació la necesidad social de saber leer y escribir, y es gracias a ésta necesidad que existen las instituciones educativas. Con el transcurso de los años y sus reformas en los diseños curriculares a éstas enseñanzas educativas se unieron los estudios de Oratoria y de la Gramática.

La morfología, la sintaxis y la fonética eran aspectos a los que se dedicaba gran parte de las acciones de las escuelas. Los currículos en el campo de lenguaje estaban marcados por un gramaticalismo y memorización de las normativas lingüísticas. Por muchos años hemos vistos los alarmantes resultados escolares en donde podemos observar que los alumnos no saben explicar sus ideas o sus interpretaciones personales, no entienden lo que leen y mucho menos pueden hacer una redacción.

El Analfabetismo Funcional nace de la Pobreza Motivacional que hace que la ineptitud se apodere de los alumnos a la hora de resolver conflictos cuando de lengua escrita hablamos haciendo que cada vez sea más difícil enseñar, aprender y desarrollar en los alumnos las competencias comunicativas básicas.

Los centros educativos no motivan a sus alumnos lo que genera una relación directa con la desmotivación de los docentes al momento de enseñar y formular estrategias de enseñanzas para cambiar los factores motivacionales intrínsecos y extrínsecos del conjunto de elementos que conforman la Instrucción Educacional del individuo como son: El estado, centros educativos, docentes, discentes, padres de familia y sociedad.

\section{Introducción}

En diciembre del 2015 se firmó la Nueva Declaración de Los Objetivos de Desarrollo Sustentables, ODS, que fue firmada por 189 países de todo el mundo con el auspicio de las Naciones Unidas y cuyo principal objetivo es comprometerse a incrementar el esfuerzo mundial para reducir los principales problemas de la humanidad como es la pobreza, sus causas y sus manifestaciones.

Dentro de los 8 Objetivos que conforman los ODS, el segundo objetivo es: Lograr la educación primaria universal.

Según La Real Academia de la Lengua (2014) define a La Pobreza como la Falta o la escasez de algo, a la Lectura, como la acción de leer. 
Entonces podemos citar algunas de las Frases de grandes escritores, tales como las siguientes:

"Los libros curan la más peligrosa de las enfermedades humanas: La ignorancia"

Un libro debe ser el hacha que rompa el mar helado que hay dentro de nosotros". Franz Kafka, escritor.

"Seríamos peores de lo que somos sin los buenos libros que leímos, más conformistas, menos insumisos y el espíritu crítico, motor del progreso, ni siquiera existiría". Mario Vargas Llosa, Premio Nobel de Literatura.

Cuando leemos desarrollamos la habilidad para comprender y saber utilizar las competencias lingüísticas requeridas o valoradas por el individuo, entiéndase por competencias lingüísticas la expresión de sentimientos, vivencias y opiniones, la participación eficaz en sus situaciones comunicativas tanto oral como escrita, la generación de empatía con otras culturas y personas, la resolución de conflictos, la apreciación de la versatilidad del lenguaje entre otras.

\section{Objetivo general}

Generar competencias mediante las Tools de la Lectura, la Lingüística y la Literatura en los discentes del milenio.

\section{Objetivos específicos}

$>$ Promover la formación de lectores mediante la pedagogía integradora.

$>$ Ampliar el horizonte cultural de los discentes

$>$ Vincular en todos los centros educativos, en todos los niveles y a en todo el mundo, la inclusión del Lenguaje, la Lingüística y la Literatura con otras formas de expresión artística en sus mallas curriculares

\section{Enseñar lengua para aprender a comunicarse y obtener éxito personal y profesional.}

La comunicación es mucho más que las palabras que emitimos. Éstas forman solamente una pequeña parte de nuestra expresividad como seres humanos.

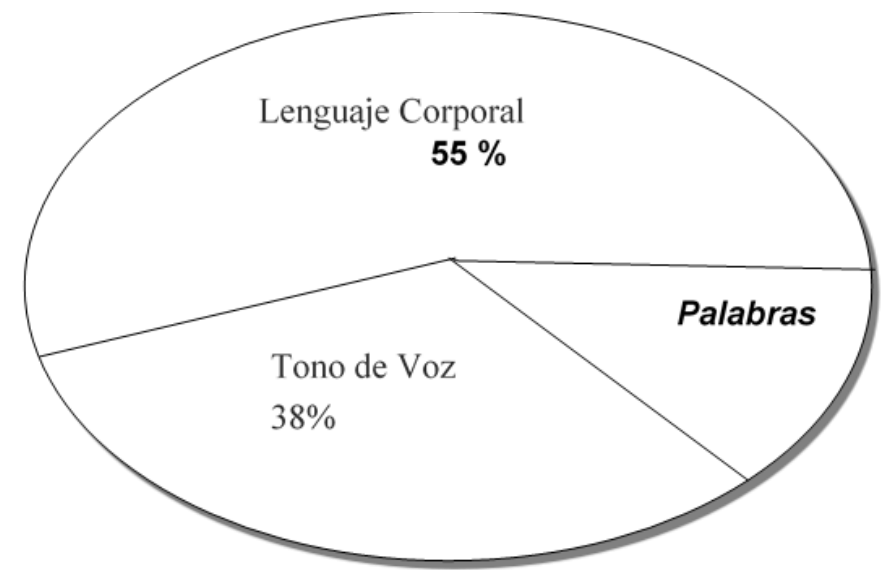


Figura 1. El 93\% de nuestra Comunicación es Lenguaje No Verbal

El diseño curricular de la materia de Lengua y Literatura debe tener como objetivo primordial la educación Lingüística, no sólo en orientarse en el conocimiento de los aspectos formales de los códigos de la lengua, sino, de que el alumno adquiera el dominio del lenguaje en sus múltiples usos, es decir, como hablante, oyente, lectores y escritores de diversos textos con naturales e intención distintos.

El Escritor y Filósofo Francis Bacon decía: "La lectura hace al hombre completo; la conversación lo hace ágil, el escribir lo hace preciso".

La brújula pedagógica debe centrarse en generar a las discentes competencias comunicativas, articulando la educación lingüística con la enseñanza exigida, previa investigación de la diversificación lingüística y sociocultural del medio en que nos desenvolvemos.

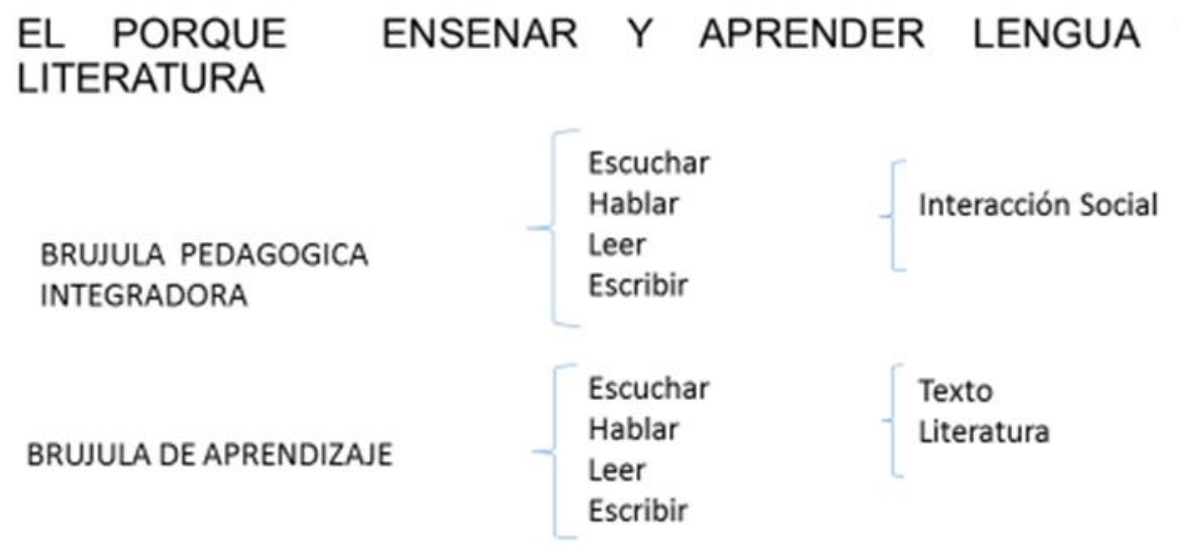

Figura 2. Por qué ensenar y aprender lengua y literatura Elaborado por: La Autora

Pero, ¿qué es el lenguaje Verbal? Es aquella cátedra que aprendemos desde los primeros años de estudios y lo representamos en el siguiente gráfico: 


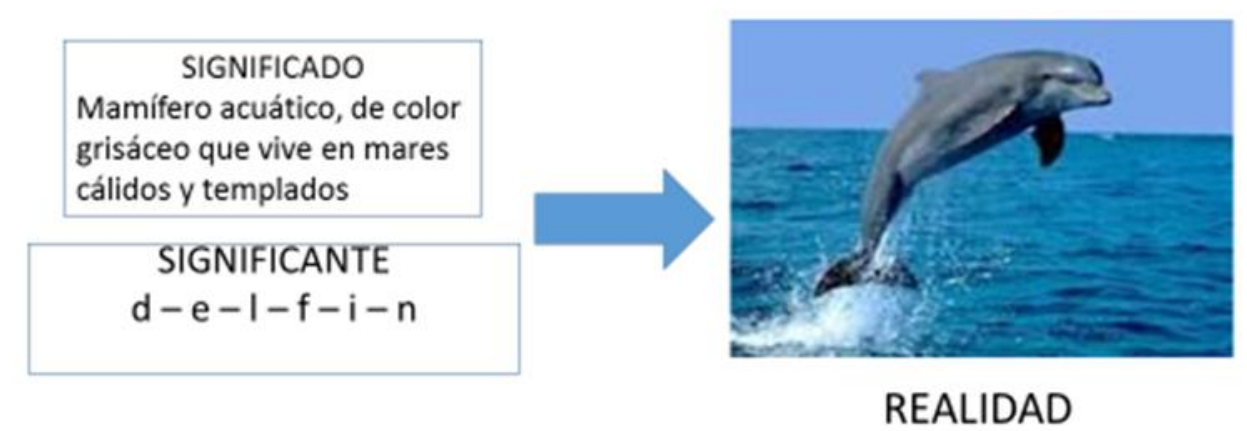
El Significante es la parte física o gráfica, en otras palabras, las letras y los sonidos, el Significado es la parte psíquica o contenido, es decir la parte léxica y lo Referente es la realidad ideal, es decir, la imagen mental que se tiene

Figura 3. El Significado. Elaborado por: La autora

\section{El lenguaje, la Literatura y la Lingüística como herramienta}

A medida que avanza a pasos agigantados el mundo y su entorno, se incrementa la necesidad imperiosa de comunicarse, necesidad esencial para la subsistencia de los individuos, utilizando el valioso recurso con el que se cuenta como es el del lenguaje hablado, escrito, y de otros recursos y medios de comunicación.

La comunicación se ha convertido en un foco de atención de las ciencias humanas, como meta de los procesos de investigación y como medio de desarrollo social, y aunque suene paradójico, dentro del boom tecnológico que en los actuales momentos nos encontramos y pese al progreso de la civilización mundial que se ha tenido, es más visible la incomunicación personal y social y sobre todo las diferentes dificultades comunicativas que subyacen en el individuo.

Es innegable que los llamados "medios modernos de comunicación”, tales como la radio, la prensa, el teléfono, el cine, la televisión, el video y el Internet le han aportado al hombre una mayor cobertura y rapidez en los procesos comunicativos; pero también es un hecho que estos procesos han resultado increíblemente complejos, entre otras razones, por la enorme y variada información que manejan, por nuevas necesidades, nuevas formas de vida y mayor complejidad de la tecnología. Pero, además, -lo que parece más grave- la comunicación tiende a ser impersonal, masiva, cuyos beneficios para el futuro humano son muy discutibles (VILLAMIZAR, 2002).

El cerebro, es un órgano del sistema nervioso del cuerpo humano, el mismo que está conformado por 3 hemisferios, que son: El hemisferio Izquierdo, el hemisferio central y el hemisferio derecho. Cada uno de estos hemisferios conglomera las competencias con las que contamos los seres humanos, que no es otra cosa que el conjunto de habilidades, conocimientos y aptitudes que sirven de herramienta para que los individuos puedan desenvolverse. 
El hemisferio Izquierdo concentra los conocimientos que posee el individuo, es decir, procesa toda la información analítica y secuencial, analiza, abstrae, mide el tiempo, cuenta, los procedimientos los planea paso a paso, verbaliza. Piensa en palabras y en números, es decir, se guía por la lógica lineal y binaria, por ejemplo: si - no, arriba - abajo, antes - después, más menos, 1, 2, 3, etc.

El hemisferio Central es el especializado en la percepción global, sintetizando la información que le llega, entendemos las metáforas, soñamos, creamos nuevas combinaciones de ideas

El hemisferio derecho se refiere a lo intuitivo en vez de lo lógico, a diferencia del Izquierdo de pensar en palabras y en números, éste piensa en imágenes, en símbolos y en sentimientos. Tiene capacidad imaginativa y fantástica, espacial y perceptiva. Este hemisferio se interesa por las relaciones, es relacional, no le preocupan las partes en sí, sino saber cómo encajan y se relacionan unas partes con otras.

Según Manlove, (2005) cree que tanto el hemisferio derecho como el hemisferio izquierdo contribuyen al procesamiento y la comprensión del lenguaje: el hemisferio izquierdo procesa tanto la semántica como la sintaxis del discurso, mientras que el hemisferio derecho procesa la emocionalidad del lenguaje, la prosodia del discurso y el lenguaje no verbal, por ejemplo, los movimientos corporales.

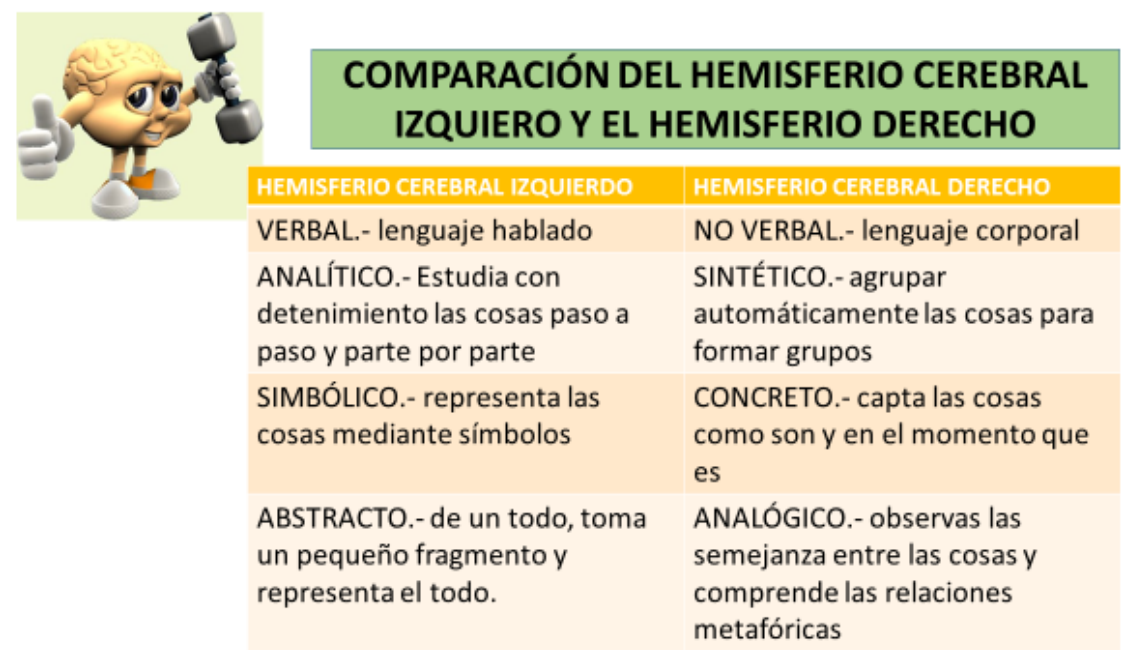

Figura 4. Comparación del hemisferio cerebral izquierdo y el hemisferio derecho Elaborado por la autora

"La lengua es el instrumento simbólico mediante el cual, como usuarios, modificamos nuestro entorno para acceder a una visión particular del mismo. Posibilita, además, la práctica lingüística, es decir, se aprende a usar la lengua para ser más efectivos en su manejo. Incluso, es una herramienta que permite la estructuración del pensamiento y la reflexión sobre sí misma para adquirirla de manera más efectiva. Por estos motivos, enseñar lengua debe aportar habilidades y conocimientos mínimos para desenvolverse en el mundo estructurado y dirigido por personas alfabetizadas. 
Además, posibilita la comunicación y, desde esta perspectiva, el desarrollo de la compete ncia comunicativa. La lengua es comunicación y eso hace que posea una dimensión social imposible de ignorar. El enfoque comunicativo plantea que la enseñanza de la lengua debe centrarse en el desarrollo de las habilidades y conocimientos necesarios para comprender y producir eficazmente mensajes lingüísticos en distintas situaciones de comunicación. Desde este enfoque, se propone enseñar la lengua partiendo de las macrodestrezas lingüísticas: hablar, escuchar, leer y escribir textos completos en situaciones comunicativas reales (CASSANY, 1997).

Los docentes tienen que hacer que los alumnos adquieran habilidades comunicativas que les permitan ser hablantes y lectores competentes, y el único camino para lograrlo es mediante una metodología de trabajo innovadora y eficaz, es decir, una pedagogía integradora, y para ello el maestro debe buscar siempre la actualización constante de sus conocimientos, que nutran e hidraten sus nuevas visiones y que alimenten su quehacer pedagógico.

La tarea colosal de todos los docentes es convertir todos los centros educativos en centros llenos de vida desde la educación básica, pues la clave del cambio de un país está en la educación y los maestros son los agentes esenciales de ese cambio.

Lo más importante en la cadena educativa de un país es la calidad de sus docentes, el verdadero hombre no mira en qué lado se vive mejor sino del lado está el deber y el deber de todos está al lado de la justicia y del bien común.

\section{Bibliografía}

CASSANY, D. (1997). Enseñar lengua, Actualización y fortalecimiento curricular de la educación básica. Editorial Grao.

Manlove, George (Febrero de 2005). Deleted Words. UMaine Today Magazine. Consultado el 9 de febrero de 2007)

VILLAMIZAR DE CAMPEROS, Y. (2002). Comunicación, lenguaje y pensamiento II. Universidad de Pamploma:Colombia. 